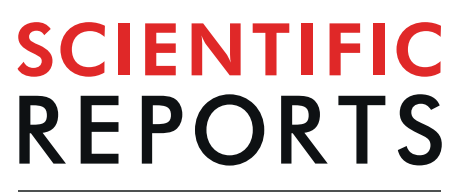

natureresearch

\title{
Glucose metabolism regulates expression of hair-inductive genes of dermal papilla spheres via histone acetylation
}

\author{
Mina Choi ${ }^{1,3}$, Yeong Min Choi ${ }^{2,3}$, Soo-Young Choi ${ }^{2}$, In-Sook An², Seunghee Bae $\mathbb{1}^{1}$, \\ Sungkwan $\mathrm{An}^{1 *}$ \& Jin Hyuk Jung $\mathbb{B}^{2 *}$
}

Cellular metabolism is one of the crucial factors to regulate epigenetic landscape in various cells including immune cells, embryonic stem cells and hair follicle stem cells. Dermal papilla cells (DP) interact with epithelial stem cells to orchestrate hair formation. Here we show that active DP exhibit robust aerobic glycolysis. We observed decrease of signature genes associated with hair induction by DP in presence of low glucose $(2 \mathrm{mM})$ and glycolysis inhibitors. Moreover, hair shaft elongation was attenuated by glycolysis inhibitors. Interestingly, excessive glucose is able to increase the expression of hair inductive genes and elongation of hair shaft. We also observed glycolysis-mediated histone acetylation is increased and chemical inhibition of acetyltransferase reduces expression of the signature genes associated with hair induction in active DP. These results suggest that glucose metabolism is required for expression of signature genes associated with hair induction. This finding may be beneficial for establishing and maintaining of active DP to generate hair follicle in vitro.

The dermal papilla (DP), specialized mesenchymal cells at the base of hair follicle, serve crucial roles in hair follicle formation ${ }^{1}$. DP secrete various factors, which initiate hair follicle formation by activating skin epithelial stem cells $^{2,3}$. Generating hair follicle in vitro is a major challenge since DP quickly lose hair-inductive activity during passaging in the conventional two-dimensional (2D) culture ${ }^{4}$. In order to restore intrinsic properties of DP, 3D sphere culture has been investigated as an alternative method. The signature genes associated with hair induction can be partially restored in DP using 3D sphere culture ${ }^{5}$. Compared to 2D, 3D sphere culture offers a more physiological relevant system where cell-cell communication as well as microenvironments is more closely represent in vivo. Cells respond to their microenvironment by stimulating various gene transcription and occupy their "niche" to differentiation ${ }^{6}$. Many lines of scientific evidence support that metabolic alteration in 3D culture towards nutrient as well as oxygen diffusion would be crucial for recapitulation of in vivo. For example, 3D cultures of human pluripotent stem cell derived cardiomyocytes express high levels of enzymes involved in OXPHOS ${ }^{7}$. And 3D cultures of HepaRG spheroids show robust activation of glucose and lipid metabolism ${ }^{8}$. Similarly, energy metabolism has been shown to be one of the major cues during hair follicle formation. Especially, lactate dehydrogenase (LDHA), an enzyme involved in anaerobic glycolysis is required for hair follicular stem cell activation ${ }^{9}$. Whereas, the relationship between cellular metabolism and functional properties of DP has been poorly understood although DP are one of the primary cells for hair follicle generation ${ }^{10}$. In this study, we determined the relationship between glucose metabolism and intrinsic properties of DP, especially expression of hair inductive genes. Because intrinsic properties of DP are restored in the sDP (sphere cultured DP) but not in the cDP (two dimensional cultured DP) according to the previous report ${ }^{4}$, we employed sDP and cDP to determine glucose metabolism.

\footnotetext{
${ }^{1}$ Research Institute for Molecular-Targeted Drugs, Department of Cosmetics Engineering, Konkuk University, Seoul, 05029, South Korea. ${ }^{2}$ Korea Institute of Dermatological Science, GeneCellPharm Corporation, 375 Munjeong 2(i)dong, Songpa-gu, Seoul, 05836, South Korea. ${ }^{3}$ These authors contributed equally: Mina Choi and Yeong Min Choi. *email: Ansungkwan@konkuk.ac.kr; Jungjh@skinreseach.or.kr
} 
a

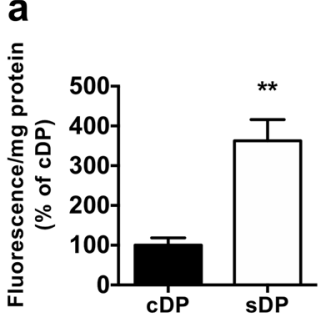

e
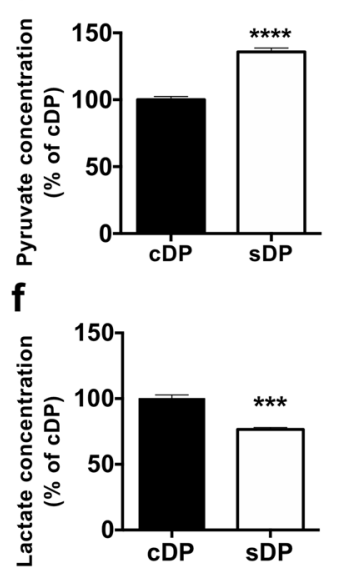

b

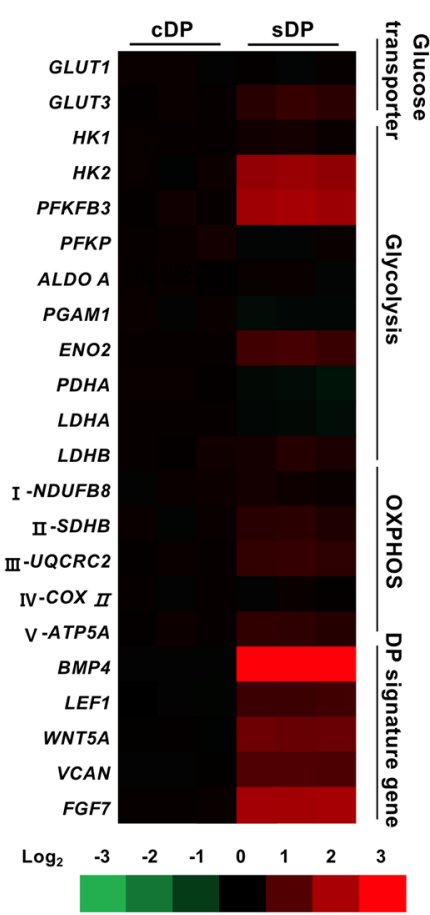

C

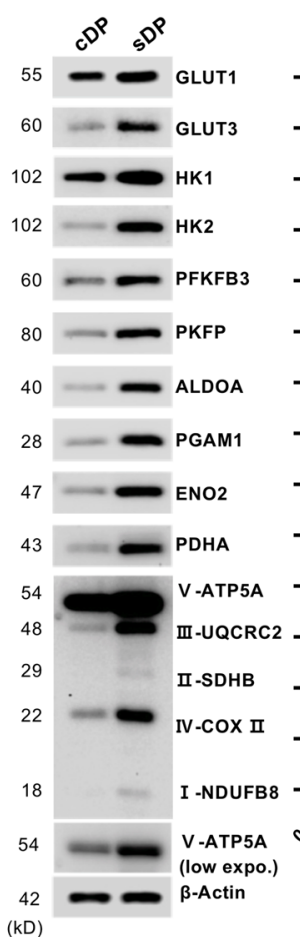

d

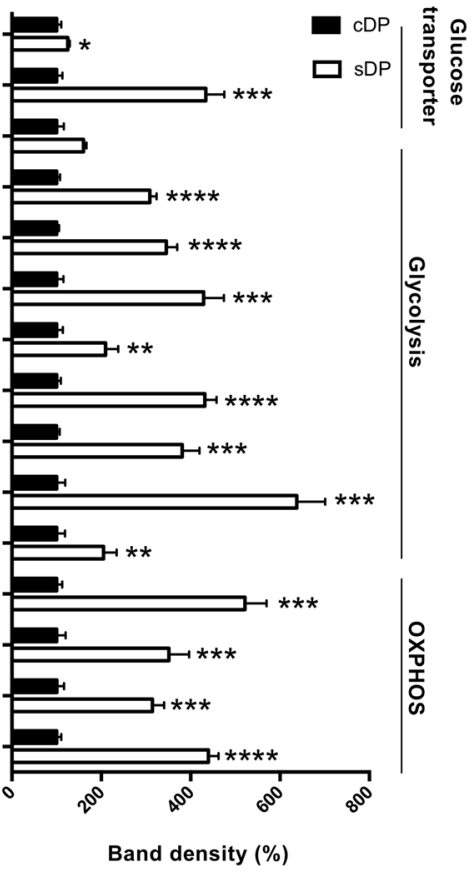

Figure 1. Glucose metabolism is activated in sDP. (a) Glucose uptake was measured by transporting of fluorescent Deoxyglucose Analog (2-NBDG) at $80 \mu \mathrm{M}$ in cultured DP (cDP) and DP spheres (sDP). (b) mRNA expression of glucose metabolism and signature gene associated with hair induction was measured in $\mathrm{CDP}$ and sDP. (c) Protein levels of metabolic enzyme were measured in cDP and sDP. (d) Quantification of western blot analysis from three independent experiments (e) Intracellular pyruvate was measured in cDP and sDP.

(f) Extracellular lactate was measured from $\mathrm{cDP}$ and sDP. Data (a,d,e) shown are mean $\pm \mathrm{STE}$ and analyzed by student t-test $(* p<0.05, * * \mathrm{p}<0.01, * * * \mathrm{p}<0.005, * * * * \mathrm{p}<0.001)$

\section{Results}

Glucose metabolism is activated in sDP. To measure glucose metabolism, we determined glucose uptake in $\mathrm{cDP}$ and sDP using fluorescent 2-NBDG. Glucose uptake was 3-folds higher in sDP compared to cDP, which indicating sDP exhibit robust glycolysis (Fig. 1a). Consequently, the levels of mRNA expression of metabolic enzymes were increased in SDP along with high expression of DP signature genes (Fig. 1b). Protein levels of metabolic enzyme were also increased in SDP (Fig. 1c,d). To determine whether sDP exhibit anaerobic or aerobic glycolysis, we measured pyruvate and lactate levels. sDP showed higher pyruvate levels but not lactate levels, which indicating aerobic glycolysis is activated by sDP (Fig. 1e,f). We also compared expression of the genes related with aerobic glycolysis between $3 \mathrm{D}$ cultured DP and paired fresh DP from microarray data (GSE44765_5 $)^{5}$. The expression levels of enzymes, which related with glycolysis and oxidative respiration were increased from 3D cultured DP (Table S1). These results indicated that glucose metabolism is induced in sDP along with high expression of hair inductive genes.

Glucose metabolism is required for the expression of hair inductive genes. Because sDP exhibit robust glycolysis, we incubated SDP in different glucose concentration to determine whether glucose metabolism is required for the expression of signature genes associated with hair induction. Expression of DP signature genes including BMP4, LEF1, WNT5A, VCAN and FGF7 were decreased from sDP in presence of low glucose (2 mM) compared with physiological of glucose $(5.5 \mathrm{mM})$ without alteration in cellular viability (Fig. $2 \mathrm{a}-\mathrm{f})$. Consequently, we used 2-deoxy-d-glucose (2DG) to determine whether inhibition of glycolysis modulates the expression of DP signature genes. 2DG treated DP showed decrease of glucose uptake and glucose-derived metabolites without alteration in cellular viability (Fig. $2 g-j$ ). The expression of the signature genes associated with hair induction was decreased by 2DG (Fig. $2 \mathrm{k}-\mathrm{o}$ ). Moreover, expression of the signature genes associated with hair induction was decreased by glycolytic inhibitors including 3BP and WZB117 (Fig. S2a-j). Interestingly, excessive glucose $(10 \mathrm{mM})$ supplement increased expression of the signature genes associated with hair induction (Fig. 2p-t). Consequently, hair shaft elongation was attenuated by glycolytic inhibitors (Fig. $2 \mathrm{u}, \mathrm{v}$ ). We also found that hair shaft elongation was enhanced by increased glucose concentration (Fig. 2w,x). These results suggest that glucose metabolism is required for expression of the genes associated with hair induction in DP.

Glycolysis-mediated acetylation is required for the expression of hair signature genes. Excessive glucose promotes hyperacetylation of histones, which directly activates the expression of genes in 

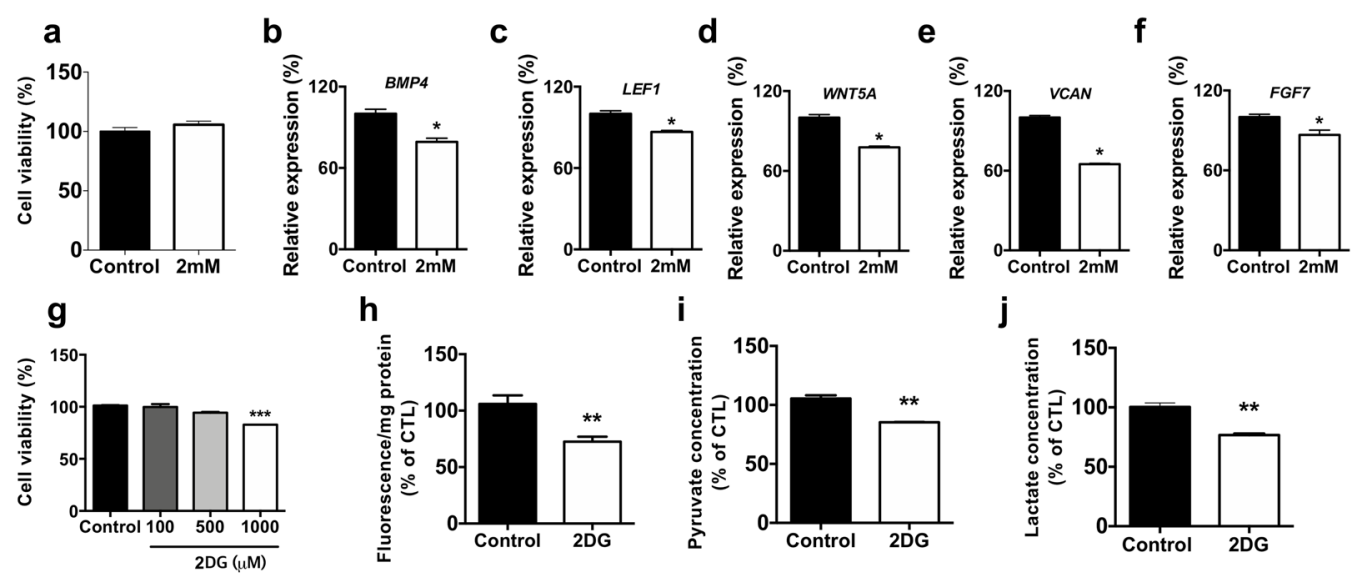

j
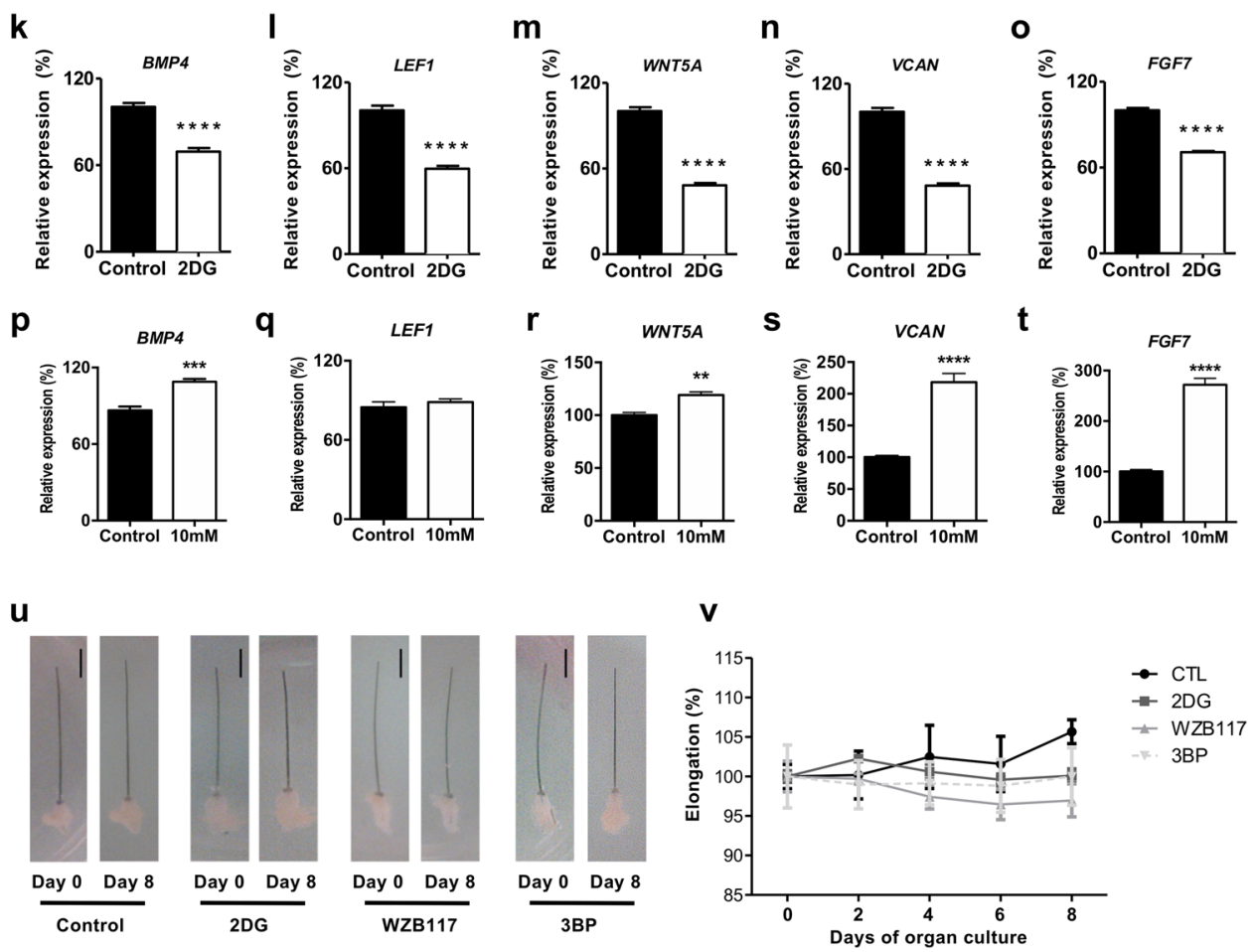

$\mathbf{W}$
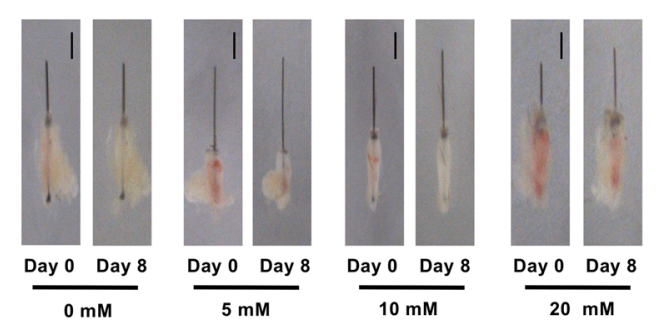

$\mathbf{x}$

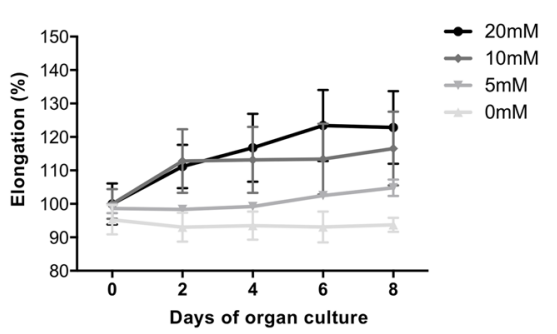

Figure 2. Glucose metabolism is required for the induction of hair inductive genes. (a) Cellular viability was measured in sDP after $48 \mathrm{~h}$ of incubation with either $5.5 \mathrm{mM}$ (control) or $2 \mathrm{mM}$ glucose incubation. (b-f) mRNA expression of indicated genes were measured in sDP after $48 \mathrm{~h}$ of incubation with either $5.5 \mathrm{mM}$ (control) or $2 \mathrm{mM}$ glucose. (g) Cellular viability was measured in sDP presence of indicated concentration of 2 deoxy-d-glucose (2DG) for $48 \mathrm{~h}$. (h) Glucose uptake was measured in sDP after $48 \mathrm{~h}$ of incubation with either vehicle or $500 \mu \mathrm{M}$ of $2 \mathrm{DG}$ treatment. (i) Intracellular pyruvate was measured in sDP after $48 \mathrm{~h}$ of incubation with either vehicle or 2DG $(500 \mu \mathrm{M})$. (j) Extracellular lactate was measured from sDP after $48 \mathrm{~h}$ of incubation with either vehicle or $2 \mathrm{DG}(500 \mu \mathrm{M})$. (k-o) mRNA expression of indicated genes were measured in sDP after $48 \mathrm{~h}$ of incubation with either vehicle or $2 \mathrm{DG}(500 \mu \mathrm{M})$. $(\mathbf{p}-\mathbf{t})$ mRNA expression of indicated genes were measured in SDP after $48 \mathrm{~h}$ of incubation with either control $(5.5 \mathrm{mM}$ glucose) or $10 \mathrm{mM}$ glucose treatment in presence of $2 \mathrm{DG}(500 \mu \mathrm{M})$. (u) Isolated mice hair follicles were treated with each indicated chemicals of $500 \mu \mathrm{M}$ of $2 \mathrm{DG}$ or $10 \mu \mathrm{M}$ of WZB 117 or $20 \mu \mathrm{M}$ of 3-Bromopyruvate (3BP) for 8 days. Images of representative mice hair 
follicle were taken from each group at day 0 and day 8 , and the length of mice follicle was measured. Scale bar $=300 \mu \mathrm{m}(\mathbf{v})$ Quantification of the hair shaft of length over time. (w) Isolated mice hair follicles were treated with each indicated glucose concentration for 8 days in presence of 2DG $(500 \mu \mathrm{M})$. Images of representative mice hair follicle were taken from each group at day 0 and day 8 , and the length of mice follicle was measured. Scale bar $=300 \mu \mathrm{m}(\mathrm{x})$ Quantification of the hair shaft length over time. Data (a-t) shown are mean \pm STE and analyzed by Student t-test $(* p<0.05, * * \mathrm{p}<0.01, * * * \mathrm{p}<0.005, * * * * \mathrm{p}<0.001)$.

nutrient-favorable conditions ${ }^{11}$. To investigate the link between glucose metabolism and the expression of genes associated hair induction in DP, we measured histone acetylation in cDP and sDP. Interestingly, the levels of histone acetylation were increased in sDP (Fig. 3a,b). Moreover, we found high expression levels of histone acetylating enzymes from DP of anagen hair follicle compared with pairing fibroblasts in web-based meta-analysis $($ GSE31324) 4 using microarray data (Table S2). Consequently, we measured histone acetylation after 2DG treatment to determine whether glucose metabolism is required for histone acetylation. The levels of histone acetylation were decreased by $2 \mathrm{DG}$ treatment (Fig. 3c,d). These results indicated that histone acetylation is regulated by glycolysis in DP. We used two histone acetyltransferase inhibitors, C646 and Ginkgolic acid to determine whether glucose-mediated histone acetylation is required for the expression of genes associated with hair induction in DP. The expression of genes associated with hair induction was decreased by these inhibitors without alteration in cellular viability as well as glucose uptake (Fig. $3 \mathrm{e}-\mathrm{k}$ ). These results suggested that DP rely on glycolysis for the expression of DP signature genes through histone acetylation.

\section{Discussion}

DP showed metabolically less active compared with neighboring cells in plucked hair according to previous report $^{12}$. Our findings suggest that active DP rely on aerobic glycolysis that differs from hair follicle stem cells, which exhibit anaerobic glycolysis ${ }^{9}$. Compartmentation of metabolism in the tissue have been reported including astrocyte-neuron metabolic cooperation ${ }^{13}$. Therefore, it would be interesting to investigate metabolic interplay between DP and hair follicle stem cells during hair cycle as well as hair regeneration. Glycolysis is required for the expression of genes associated with hair induction (Fig. 2). Carbohydrates including galactose, glucosamine and fructose are transported through glucose transporters and utilized in glucose metabolism ${ }^{14,15}$. Therefore, excessive carbohydrates-mediated DP inductivity should be determined in the future. On the other hands, glycolysis-mediated histone acetylation promotes differentiation of embryonic stem cells and proinflammatory cytokine production in helper T cells through epigenetic control ${ }^{16,17}$. Based on these scientific backgrounds, we hypothesized that the incorporated excessive glucose into sDP promotes signature gene transcription by histone acetylation. We observed high levels of histone acetylation in sDP (Fig. 3a,b). Moreover, histone acetylating enzymes including KAT6A and KAT6B were increased in DP from anagen hair follicles (Table S2). Glycolysis inhibitor, 2DG inhibits histone acetylation in sDP (Fig. 3c,d). Our data suggest glucose is required for histone acetylation, which is important for the expression of hair inductive genes (Fig. 3). Interestingly, our data correlates with recent studies that histone deacetylase inhibitors including tricostatin A (TSA) and valproic acid promote hair inductivity ${ }^{18,19}$. Taken together, our finding suggests the standpoint where we could see a novel metaboloepigenetic regulation of DP although fine tuning of histone acetylation and epigenetic regulation of DP signature genes would be remains to explore in the future.

\section{Methods}

All methods were performed in accordance with the relevant guidelines and regulations

Cell culture and reagents. Dermal papilla cells (DP; PromoCell GmbH, Heidelberg, Germany, Lot 325Z017.1 and 322Z030.1) were maintained in the Follicle Dermal Papilla Cell Basal Medium (PromoCell $\mathrm{GmbH}$ ) supplemented with $4 \mu \mathrm{l} / \mathrm{ml}$ bovine pituitary extract, $0.04 \mathrm{ml} / \mathrm{ml}$ fetal calf serum, $1 \mathrm{ng} / \mathrm{ml}$ basic recombinant human fibroblast growth factor, $5 \mu \mathrm{g} / \mathrm{ml} \mathrm{recombinant} \mathrm{human} \mathrm{insulin} \mathrm{(PromoCell).} \mathrm{Cells} \mathrm{were} \mathrm{cultured}$ with $50 \mathrm{mg} / \mathrm{ml}$ primocin (Invivogen, Toulouse, France, \#ant-pm-2) in a humidified $5 \% \mathrm{CO}_{2}$ incubator at $37^{\circ} \mathrm{C}$. $\mathrm{cDP}$ and sDP were maintained as previously reported ${ }^{4}$. Briefly, cDP were maintained in $60 \mathrm{~mm}$ dish (SPL, Gyeonggi-do, Korea, \#20060) and sDP were maintained in Ultra Low Attachment Culture Dish (Corning, New York, USA \#3261). Both cDP and sDP were maintained same DP media described above. 2-deoxy-d-glucose (2DG), WZB117 and Bromopyruvic acid (3BP) were purchased from Sigma-Aldrich (St. Louis, USA, \#D8375, \#SML0621, \#16490 respectively). D-(+)-Glucose powder were purchased from Sigma-Aldrich (\#G7021). C646 (\#10549) and Ginkgolic acid (\#18422) were purchased from Cayman Chemical (Ann Arbor, USA).

Cell viability assay. The viability of the DP was measured using the water-soluble tetrazolium salt (WST-1) assay (EZ-Cytox Cell Viability Assay Kit; ITS-Bio, Seoul, Korea, \# EZ3000) according to previously described ${ }^{20}$. Absorbance was determined at $450 \mathrm{~nm}$ using an iMark microplate reader (Bio-Rad laboratories, Hercules, USA).

2-deoxy-d-glucose ransport Assay. DP were cultured for $48 \mathrm{~h}$ with $80 \mu \mathrm{M}$ of the fluorescent 2-deoxy-d-glucose analog, 2-NBDG (2-deoxy-2-[(7-nitro-2,1,3- benzoxadiazol-4-yl)amino]-D-glucose, Cayman, \#186689-07-6) in glucose free media for $30 \mathrm{~min}$. Reactions were terminated by solubilizing cells according to previously described ${ }^{14}$. Briefly, 2-NBDG fluorescence was measured at $485 / 538 \mathrm{~nm}$ (excitation/emission) and fluorescent values were normalized to the respective protein concentration using BCA protein Assay kit (Thermo Fisher Scientific, Rockford, USA, \#23225). 


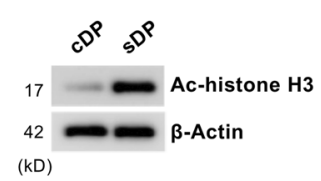

e

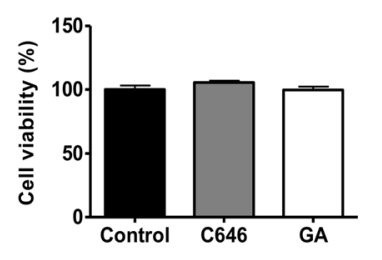

b

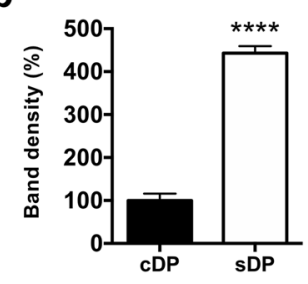

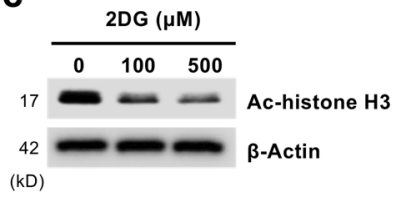

d

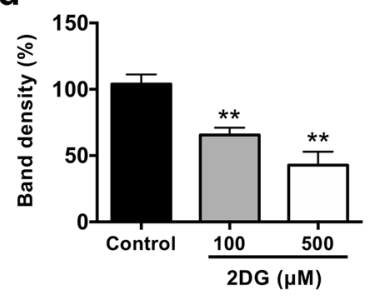

g

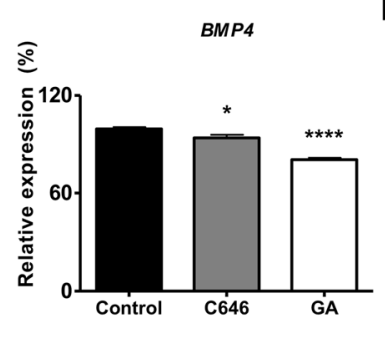

h

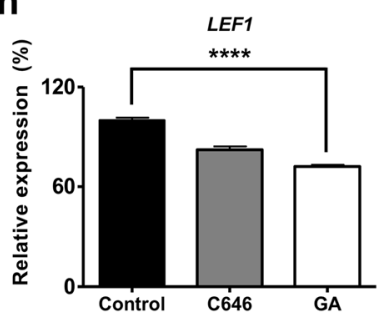

i

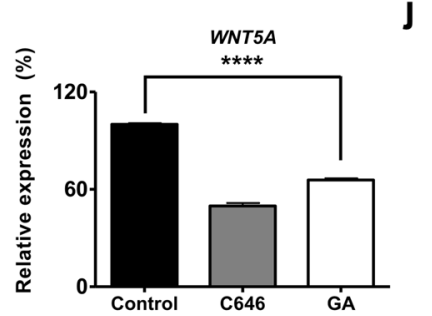

j

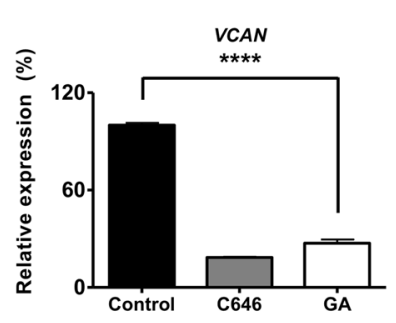

k

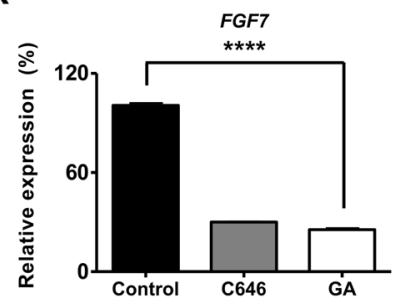

Figure 3. Glycolysis-mediated acetylation is required for the expression of hair signature genes (a) Protein levels of acetylated histone were measured in cDP and sDP. (b) Quantification of western blots from three independent experiments. (c) Protein level of acetylated histone was measured in sDP after $48 \mathrm{~h}$ of incubation with indicated concentration of 2DG. (d) Quantification of western blots from three independent experiments. (e) Cellular viability was measured in sDP after $48 \mathrm{~h}$ of incubation with either vehicle or $10 \mu \mathrm{M}$ C646 or $10 \mu \mathrm{M}$ Ginkgolic acid. (f) Glucose uptake was measured in sDP after $48 \mathrm{~h}$ of incubation with either vehicle or $10 \mu \mathrm{M}$ C646 or $10 \mu \mathrm{M}$ Ginkgolic acid. (GA). (g-k) mRNA expression of indicated genes were measured in sDP after $48 \mathrm{~h}$ of incubation with either vehicle or $10 \mu \mathrm{M}$ C646 or $10 \mu \mathrm{M}$ Ginkgolic acid (GA). Data shown are mean \pm STE and analyzed by one-way analysis of variance (ANOVA) $(* p<0.05, * * p<0.01, * * * * p<0.001)$.

Quantitative real-time PCR analysis. Total cellular RNA was isolated from DP using TRIzol reagent (Thermo Fisher Scientific, \# 15596-018) and cDNA was synthesized using M-MLV reverse transcriptase (Thermo Fisher Scientific, \#28025-013) according to previously decribed ${ }^{21}$. Quantitative reverse transcription polymerase chain reaction analysis was performed using SYBR ${ }^{\mathrm{TM}}$ green PCR master mix (Thermo Fisher Scientific, \#4309155) with a Step OnePlus Real-Time PCR System (Thermo Fisher Scientific). The sequences of the primer sets are listed in Table S3. mRNA levels were normalized to human $18 \mathrm{~S}$ rRNA. Data shown are mean \pm STE and analyzed by Student t-test $(* p<0.05)$.

Western blot analysis. Western blot analysis was performed as previously described ${ }^{22}$. Sources and working dilutions of antibodies are shown in Table S4. Each protein was detected using enhanced chemiluminescence (Bio-Rad laboratories, \#1705061) and visualized using the ChemiDoc Touch Imaging System (Bio-Rad laboratories).

Pyruvate and Lactate assays. Concentrations of intracellular pyruvate and extracellular L-lactate were measured fluorometrically (ex $544 \mathrm{~nm}$, em $590 \mathrm{~nm}$ ) using kits from Cayman Chemical (\#700470, \#700510 respectively) according to previously described ${ }^{14}$ and fluorescent values were normalized to total protein for each well determined using BCA protein Assay kit (Thermo Fisher Scientific, \#23225).

Hair shaft elongation assay. Hair shaft elongation assay was performed according to previously described $^{23-25}$. For organ cultures of mouse vibrissa follicles, individual hair follicles were dissected from the upper lip pad of 5-week-old female C57BL/6 mice. Vibrissa follicles were placed individually in 24-well plates containing Follicle Dermal Papilla Cell Basal Medium (PromoCell GmbH) supplemented with $4 \mu \mathrm{l} / \mathrm{ml}$ bovine pituitary extract, $0.04 \mathrm{ml} / \mathrm{ml}$ fetal calf serum, $1 \mathrm{ng} / \mathrm{ml}$ basic recombinant human fibroblast growth factor, $5 \mu \mathrm{g} /$ $\mathrm{ml}$ recombinant human insulin (PromoCell) with $1 \mathrm{x}$ penicillin- streptomycin solution (Gibco; Invitrogen, Carlsbad, CA, USA, \#15140-122) and each chemical was added to the culture medium. Protocols approved by the Institutional Animal Care and Use Committee (Konkuk University, Republic of Korea). No. KU16199 
Web-based meta-analysis. Microarray datasets from studies (GSE44765_5 5 and GSE22623722 ${ }^{4}$ ) were analyzed using GEO2R (https://www.ncbi.nlm.nih.gov/geo/geo2r) to determine enzyme expression of aerobic glycolysis and histone acetylation.

Ethics approval and consent to participate. All animals were care for by using protocols approved by the Institutional Animal Care and Use Committee (Konkuk University, Republic of Korea). No. KU16199.

Statistical analysis. Data were analyzed by two-tailed Student t-test, one-way analysis of variance (ANOVA) using GraphPad Prism software v.5 Unless indicated otherwise, all experiments were performed using triplicate culture dishes and data shown are the mean \pm SEM. Two-group comparisons were analyzed by two-sided Student's $t$ test. $p$ values were calculated, and $\mathrm{p}<0.05$ was considered significant.

\section{Data availability}

All study data are available from the corresponding author upon request.

Received: 7 November 2019; Accepted: 2 March 2020;

Published online: 17 March 2020

\section{References}

1. Driskell, R. R., Giangreco, A., Jensen, K. B., Mulder, K. W. \& Watt, F. M. Sox2-positive dermal papilla cells specify hair follicle type in mammalian epidermis. Dev. 136, 2815-2823, https://doi.org/10.1242/dev.038620 (2009).

2. Rendl, M., Polak, L. \& Fuchs, E. BMP signaling in dermal papilla cells is required for their hair follicle-inductive properties. Genes. Dev. 22, 543-557, https://doi.org/10.1101/gad.1614408 (2008).

3. Zhang, H. et al. iTRAQ-Based Quantitative Proteomic Comparison of Early- and Late-Passage Human Dermal Papilla Cell Secretome in Relation to Inducing Hair Follicle Regeneration. PLoS One 11, e0167474, https://doi.org/10.1371/journal. pone.0167474 (2016).

4. Ohyama, M., Kobayashi, T., Sasaki, T., Shimizu, A. \& Amagai, M. Restoration of the intrinsic properties of human dermal papilla in vitro. J. Cell Sci. 125, 4114-4125, https://doi.org/10.1242/jcs.105700 (2012).

5. Higgins, C. A., Chen, J. C., Cerise, J. E., Jahoda, C. A. \& Christiano, A. M. Microenvironmental reprogramming by threedimensional culture enables dermal papilla cells to induce de novo human hair-follicle growth. Proc. Natl Acad. Sci. USA 110, 19679-19688, https://doi.org/10.1073/pnas.1309970110 (2013).

6. Simon, M. C. \& Keith, B. The role of oxygen availability in embryonic development and stem cell function. Nat. Rev. Mol. Cell Biol. 9, 285-296, https://doi.org/10.1038/nrm2354 (2008).

7. Correia, C. et al. 3D aggregate culture improves metabolic maturation of human pluripotent stem cell derived cardiomyocytes. Biotechnol. Bioeng. 115, 630-644, https://doi.org/10.1002/bit.26504 (2018).

8. Takahashi, Y. et al.3D spheroid cultures improve the metabolic gene expression profiles of HepaRG cells. Biosci Rep 35, https://doi. org/10.1042/BSR20150034 (2015).

9. Flores, A. et al. Lactate dehydrogenase activity drives hair follicle stem cell activation. Nat. Cell Biol. 19, 1017-1026, https://doi. org/10.1038/ncb3575 (2017).

10. Driskell, R. R., Clavel, C., Rendl, M. \& Watt, F. M. Hair follicle dermal papilla cells at a glance. J. Cell Sci. 124, 1179-1182, https://doi. org/10.1242/jcs.082446 (2011).

11. Sebastian, C. \& Mostoslavsky, R. The Various Metabolic Sources of Histone Acetylation. Trends Endocrinol. Metab. 28, 85-87, https://doi.org/10.1016/j.tem.2016.11.001 (2017).

12. Williams, R., Philpott, M. P. \& Kealey, T. Metabolism of freshly isolated human hair follicles capable of hair elongation: a glutaminolytic, aerobic glycolytic tissue. J. Invest. Dermatol. 100, 834-840, https://doi.org/10.1111/1523-1747.ep12476744 (1993).

13. Belanger, M., Allaman, I. \& Magistretti, P. J. Brain energy metabolism: focus on astrocyte-neuron metabolic cooperation. Cell Metab. 14, 724-738, https://doi.org/10.1016/j.cmet.2011.08.016 (2011).

14. Jung, J. H., Iwabuchi, K., Yang, Z. \& Loeken, M. R. Embryonic Stem Cell Proliferation Stimulated By Altered Anabolic Metabolism From Glucose Transporter 2-Transported Glucosamine. Sci. Rep. 6, 28452, https://doi.org/10.1038/srep28452 (2016).

15. Mueckler, M. \& Thorens, B. The SLC2 (GLUT) family of membrane transporters. Mol. Asp. Med. 34, 121-138, https://doi. org/10.1016/j.mam.2012.07.001 (2013).

16. Moussaieff, A. et al. Glycolysis-mediated changes in acetyl-CoA and histone acetylation control the early differentiation of embryonic stem cells. Cell Metab. 21, 392-402, https://doi.org/10.1016/j.cmet.2015.02.002 (2015).

17. Peng, M. et al. Aerobic glycolysis promotes T helper 1 cell differentiation through an epigenetic mechanism. Sci. 354, 481-484, https://doi.org/10.1126/science.aaf6284 (2016).

18. Guo, L. et al. TSA restores hair follicle-inductive capacity of skin-derived precursors. Sci. Rep. 9, 2867, https://doi.org/10.1038/ s41598-019-39394-w (2019).

19. Lee, S. H. et al. Valproic acid induces hair regeneration in murine model and activates alkaline phosphatase activity in human dermal papilla cells. PLoS One 7, e34152, https://doi.org/10.1371/journal.pone.0034152 (2012).

20. Jung, J. H. et al. Triad 1 induces apoptosis by p53 activation. FEBS Lett. 584, 1565-1570, https://doi.org/10.1016/j.febslet.2010.03.011 (2010).

21. Choi, M. et al. E3 ligase RCHY1 negatively regulates HDAC2. Biochem. Biophys. Res. Commun. 521, 37-41, https://doi.org/10.1016/j. bbrc.2019.10.049 (2020).

22. Jung, J. H., Wang, X. D. \& Loeken, M. R. Mouse embryonic stem cells established in physiological-glucose media express the high KM Glut2 glucose transporter expressed by normal embryos. Stem Cell Transl. Med. 2, 929-934, https://doi.org/10.5966/sctm.20130093 (2013).

23. Robinson, M., Reynolds, A. J. \& Jahoda, C. A. Hair cycle stage of the mouse vibrissa follicle determines subsequent fiber growth and follicle behavior in vitro. J. Invest. Dermatol. 108, 495-500, https://doi.org/10.1111/1523-1747.ep12289730 (1997).

24. Jindo, T., Imai, R., Takamori, K. \& Ogawa, H. Organ culture of mouse vibrissal hair follicles in serum-free medium. J. Dermatol. 20, 756-762, https://doi.org/10.1111/j.1346-8138.1993.tb01379.x (1993).

25. Lee, J., Wu, W. \& Kopan, R. Murine vibrissae cultured in serum-free medium reinitiate anagen. J. Invest. Dermatol. 128, 482-485, https://doi.org/10.1038/sj.jid.5701024 (2008).

\section{Acknowledgements}

This work is supported by GeneCellPharm Corporation. We thank Yeon-Seung Choi for assisting histology analysis. 


\section{Author contributions}

Y.M.C., S.B., I.S.A. and J.H.J. designed the experiments and analyzed the data. M.C. and S.Y.C., performed the experiments. J.H.J and S.A. wrote the manuscript.

\section{Competing interests}

The authors declare no competing interests.

\section{Additional information}

Supplementary information is available for this paper at https://doi.org/10.1038/s41598-020-61824-3.

Correspondence and requests for materials should be addressed to S.A. or J.H.J.

Reprints and permissions information is available at www.nature.com/reprints.

Publisher's note Springer Nature remains neutral with regard to jurisdictional claims in published maps and institutional affiliations.

(c) (i) Open Access This article is licensed under a Creative Commons Attribution 4.0 International License, which permits use, sharing, adaptation, distribution and reproduction in any medium or format, as long as you give appropriate credit to the original author(s) and the source, provide a link to the Creative Commons license, and indicate if changes were made. The images or other third party material in this article are included in the article's Creative Commons license, unless indicated otherwise in a credit line to the material. If material is not included in the article's Creative Commons license and your intended use is not permitted by statutory regulation or exceeds the permitted use, you will need to obtain permission directly from the copyright holder. To view a copy of this license, visit http://creativecommons.org/licenses/by/4.0/.

(c) The Author(s) 2020 